\title{
Novel Neuropathological Features of a Child with an Epileptic Encephalopathy Due to Presumed Polyol Dysmetabolism
}

\author{
Eric David Marsh ${ }^{1}$ Anna Gunhild Christina Bergqvist ${ }^{1}$ \\ Nicholas Konstantinos Gonatas ${ }^{3, *}$ Ralph C. Eagle Jr. ${ }^{4}$ \\ ${ }^{1}$ Division of Neurology, Department of Pediatric and Neurology, Children's \\ Hospital of Philadelphia and Perelman School of Medicine at the University \\ of Pennsylvania, Philadelphia, Pennsylvania, United States \\ 2 Department of Pathology, Hackensack Hospital, Hackensack, New \\ Jersey, United States \\ ${ }^{3}$ Division of Neuropathology, Perelman School of Medicine at the \\ University of Pennsylvania, Philadelphia, Pennsylvania, United States \\ ${ }^{4}$ Department of Pathology, Wills Eye Hospital, Philadelphia, \\ Pennsylvania, United States \\ ${ }^{5}$ Department of Pathology-Neuropathology, Children's Hospital of \\ Philadelphia and Perelman School of Medicine at the University of \\ Pennsylvania, Philadelphia, Pennsylvania, United States \\ J Pediatr Epilepsy 2018;7:142-147.
}

Livija Medne $^{1}$ Patrice Hassoun ${ }^{2}$

\author{
Abstract \\ Keywords \\ - polyol disorder \\ - epileptic \\ encephalopathy \\ - pathology
}

Disorders of carbohydrate metabolism resulting in accumulation of polyols in body fluids were first described in the early 1990s by van der Knapp et al, but this diagnosis remains rare with very few patients reported with primary neurological phenotypes. We present a child with a presumed polyol disorder whose clinical course was similar to two of the original five patients and who had similar findings in quantification of urinary polyols. In addition, we document details of central nervous system pathology in this rare metabolic disorder. The basic pathology is that of a neurodegenerative disorder with hypomyelination leukodystrophy consequent to a defect of oligodendroglia and resultant axonal and neuronal loss.

\section{Introduction}

Polyols are common metabolites of carbohydrates that are generated in all body tissue, but are found in higher concentration in brain than in other organs. ${ }^{1}$ Polyols are formed by the reduction of sugars, particularly the mono and disaccharides. Most polyols are formed either from the direct reduction of glucose and galactose or from pentose sugars that are produced from the pentose phosphate shunt. The role and metabolism of polyols are unclear. To date, only a few enzymes have been shown to be important in their

\footnotetext{
Dr. Gonatas is deceased.
}

metabolism and several membrane-spanning proteins are believed to be involved in their transport. ${ }^{1,2}$ Mutations in eight enzymes have been linked to human disease. These include ribose-5-phosphate isomerase deficiency, enzymesassociated galactosemia, transaldolase deficiency, sorbitol dehydrogenase, L-arabitol dehydrogenase, and sedoheptulokinase. ${ }^{1,3-8}$ Defects in any of these enzymes are rare, with galactosemia being the most common and presents in infancy with lethargy, vomiting, diarrhea, and jaundice. ${ }^{3}$ The others cause a variety of symptoms, most of which are due to accumulation of the polyol in tissue resulting in damage to liver, gastrointestinal, retina, and both peripheral and central nervous system (CNS).

Copyright (c) 2018 by Georg Thieme Verlag KG, Stuttgart · New York
DOI https://doi.org/ 10.1055/s-0039-1678560. ISSN 2146-457X. 
Polyols can be elevated in disease processes with deranged carbohydrate metabolism such as diabetic ketoacidosis and galactosemia. The presence of elevated polyols has been most closely associated with diabetic peripheral neuropathy and retinopathy, ${ }^{9,10}$ but has been less frequently associated with primary liver or neurological disorders including multiple sclerosis. ${ }^{3,7,11}$ The mechanism of tissue changes related to polyols in diabetics has not been well elucidated. ${ }^{9,10,12}$ A few studies have also described elevation of myo-inositol and other polyols in children with trisomy $21^{13}$ and affective disorders. ${ }^{14}$ Because of emerging evidence of the potential effect of polyols in white matter of CNS, van der Knapp et al began studying the polyol body fluid concentration of patients referred to their center with undiagnosed leukodystrophies. ${ }^{8,15,16}$ From their investigations, five patients were described, each with somewhat different clinical presentations. As only five patients have been reported with primary CNS-related polyol abnormalities in over 18 years, these disorders are apparently extremely rare.

As a rare and currently untreatable disorder, evaluation of polyols in patients with profound cognitive disabilities and early onset seizures is not routinely performed. We present a new case of a possible polyol disorder that is similar to the two cases of neonatal onset epilepsy and profound developmental delay that were reported by Huck et al. ${ }^{16}$ In addition to presenting the clinical case and imaging data, we document the first description of unique pathological findings in a child with profound epileptic encephalopathy from a suspected polyol disorder.

\section{Results}

Case History: The patient was born at 35 weeks gestation by C-section to a 25-year-old mother. A previous pregnancy had resulted in a fetal demise (female fetus) at 37 weeks; no autopsy was done. Parents were not consanguineous and there was an older healthy brother. On day 1 of life, the baby developed apneic seizures and required ventilatory support. The seizures accelerated into status epilepticus. She failed numerous antiepileptic drugs (phenobarbital, phenytoin, levetiracetam, topiramate, zonisamide, vitamin B6, pyridoxal phosphate and folinic acid) and the seizures were not controlled until a ketogenic diet was started at 2 weeks of life. It was gradually advanced to a 3:1 ratio (daily grams of fat:grams of carbohydrate and protein).

Three months later while on a 4:1 ratio, she stopped seizing for a month before daily seizures returned. The seizures changed into brief versive seizures of eye blinking and head deviation without apnea. Her electroencephalographies (EEGs) were always abnormal, beginning with multifocal discharges but becoming hypsarrhythmic with electrodecrements in association with clinical spasms. Multifocal partial seizures were also captured on EEG. Further trials with steroids, gabapentin, rufinamide, oxcarbazepine, and lamotrigine were unsuccessful. She was managed for most of her life on a combination of phenobarbital, phenytoin, vigabatrin, B-6, and the ketogenic diet.
On physical examination, she was normocephalic at birth, but became increasingly microcephalic over time. She had short upper extremities and ulnar deviation of her hands. Her palate was highly arched, but she was otherwise nondysmorphic. There was no organomegaly or neurocutaneous stigmata. Over time, she developed contractures, spasticity of the extremities, low axial tone, and kyphoscoliosis. During her life, she never gained any significant developmental milestones and remained in an infantile state. She died from a respiratory illness at age five.

\section{Diagnostic Studies}

Search for an explanation of this profound epileptic encephalopathy included extensive neurometabolic, muscle, and single gene testing all of which were essentially normal. Hypothyroidism was diagnosed on Neogen screen. Magnetic resonance imaging (MRI) at 1 month of age initially suggested cortical migrational disorder (- Fig. 1A-C-white arrow) with cortical thickening and thin corpus callosum (-Fig. 1C-arrows), but these were not seen on later imaging ( - Fig. 1D-F). Instead, progressive decrease in white matter volume was noted (-Fig. 1D-E; * in white matter). Magnetic resonance spectroscopy showed a decrease in $\mathrm{N}$-acetylaspartate suggesting neuronal loss; lactate was never elevated (images not shown).

Urine polyols were mildly abnormal when first tested at age 2 (reported in units of $\mathrm{mmol} / \mathrm{mol}$ creatinine): arabitol 123.1 (32-88), erythritol 11.9 (55-192), ribitol 10.9 (8-24), xylitol 9 (5-49), and galactitol 1.8 (3-81). Further polyol testing when this child was 4 years old showed that urine polyols were now significantly elevated: arabitol 225 (32-88), erythritol 86 (55-192), ribitol 16 (8-24), xylitol 157 (5-49), sedoheptulose $0(<1)$, sedohepitol $0(<1)$. A diagnosis of suspected polyol disorder was made.

\section{Postmortem Examination}

The postmortem examination was done at the local hospital by Dr. Hassoun. The brain, spinal cord, eyes, and muscle were placed in formalin and transported to The Children's Hospital of Philadelphia. Tissues were embedded in paraffin and stained with hematoxylin and eosin, by the Klver technique (cresyl violet-luxol fast blue) for the evaluation of myelin. Bodian silver stains for axons and neurons, and for expression of various antigens by use of immunoperoxidase procedures. Three antibodies were used including neurofilament protein (NFP; Invitrogen, Waltham, MA, United States), glial fibrillary acidic protein (GFAP; Dako, Santa Clara, CA, United States), and CD163 (for macrophages; Vector, Burlingame, CA, United States).

In addition, specimens of cerebral and cerebellar white matter were post-fixed in osmium tetraoxide for ultrastructural study. The eyes were sent to Dr. Eagle.

\section{Pathological Findings}

The brain was severely atrophic (expected unfixed weight $1214 \mathrm{~g}$; actual fixed weight $811 \mathrm{~g}$ ) and had a dolichocephalic configuration (-Fig. 2A). Optic nerves and chiasm were small 

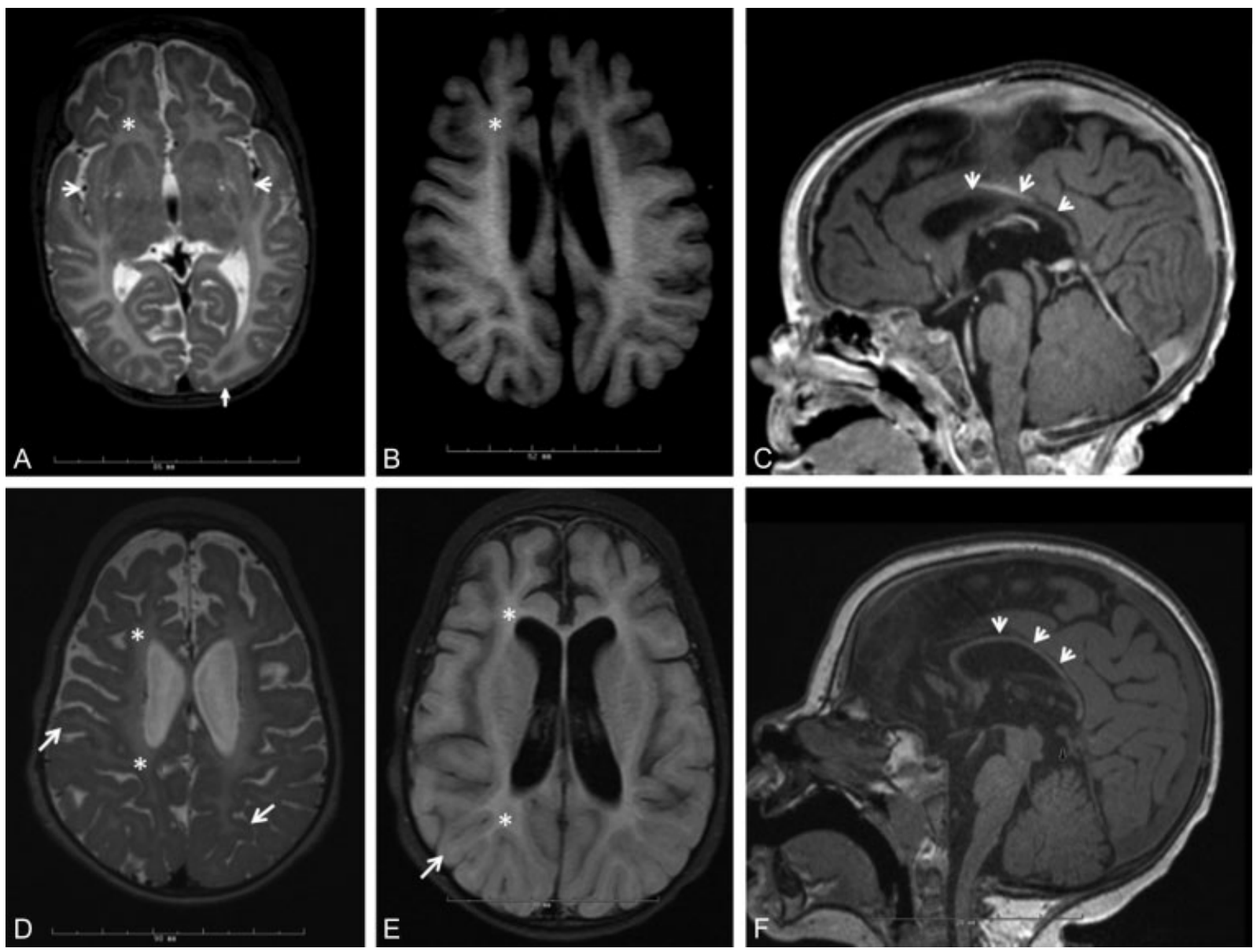

Fig. 1 Magnetic resonance imaging of the brain at 1 month and 2 years of age. For both ages, representative T2 axial (A, D), T2 flair (B, E), and T1 sagittal (C, F) images are shown. Asterisks and arrows show changes in white matter volume and signal (A, B, D, E). Atrophy of brain with enlarged ventricles and sulci is present. Thin corpus callosum is observed at both ages (C, F).

and gray. Cerebellum and brain stem were also small and folia were sclerotic. Cerebral gyral pattern was normal and the cortical ribbon was of normal thickness. There was a striking reduction in volume of white matter throughout the hemispheres, particularly in the centrum semiovale and corpus callosum ( - Fig. 2A, circle and arrow). The internal capsule appeared more normal in thickness ( - Fig. 2A, arrowhead). Deep gray nuclei and hippocampi were recognizable (-Fig. 2A, inside internal capsule). Cerebral peduncles and descending cortical tracts in pons, medulla, and spinal cord were smaller than normal (not shown). Cerebellar white matter was relatively greater in volume than cerebral white matter (not shown).

The microscopic abnormalities were unusual and did not fit a pattern of any previously described gray or white matter disorders. The findings (-Fig. $\mathbf{2 B}-\mathbf{H}$ ) included changes throughout the neuroaxis. Most prominently, were the cerebral alterations in both gray and white matter structures. There was severe neuronal loss of pyramidal and dentate cells of hippocampus, subiculum, and presubiculum with associated severe gliosis ( - Fig. 2B). There were apical dendrites present on cortical pyramidal cells by NFP staining ( $\mathbf{- F i g}$. 2C). In the white matter, there were vanishingly few myelinated fibers and diffuse hypocellularity (-Fig. 2D-F from corpus callo- sum). The majority of cells appeared to be oligodendroglia with no obvious astrocytic proliferation (no gliosis as evidenced by GFAP, - Fig. 2G) or macrophage response (as evidence by CD163 marker, - Fig. 2H) with some regions displaying a spongy state (-Fig. 2, Klüver stain [2E], and Bodian stain $[2 F])$. There was a marked paucity of axons throughout the white matter ( - Fig. 2F). The remaining myelinated fibers were highlighted by Klüver stain (-Fig. 2E).

In the deep gray structures, there was poorly defined architecture of thalamic nuclei and basal ganglia. In the cerebellum, a chronic loss of Purkinje and basket cells unassociated with Bergmann gliosis was observed. Finally, in the spinal cord, dystrophic axons were present in both Clarke's column and nucleus gracilis.

There was no obvious muscle pathology. Both eyes displayed atrophy of the retinal ganglion cell and nerve fiber layers. Use of stains for axons and myelin showed almost no axons or myelin sheaths in the optic nerves. Cataracts were not identified (not shown).

\section{Ultrastructural Features}

Electron microscopy of sections from the white matter showed paucity of myelinated axons ( - Fig. 3A-C). The majority of the 

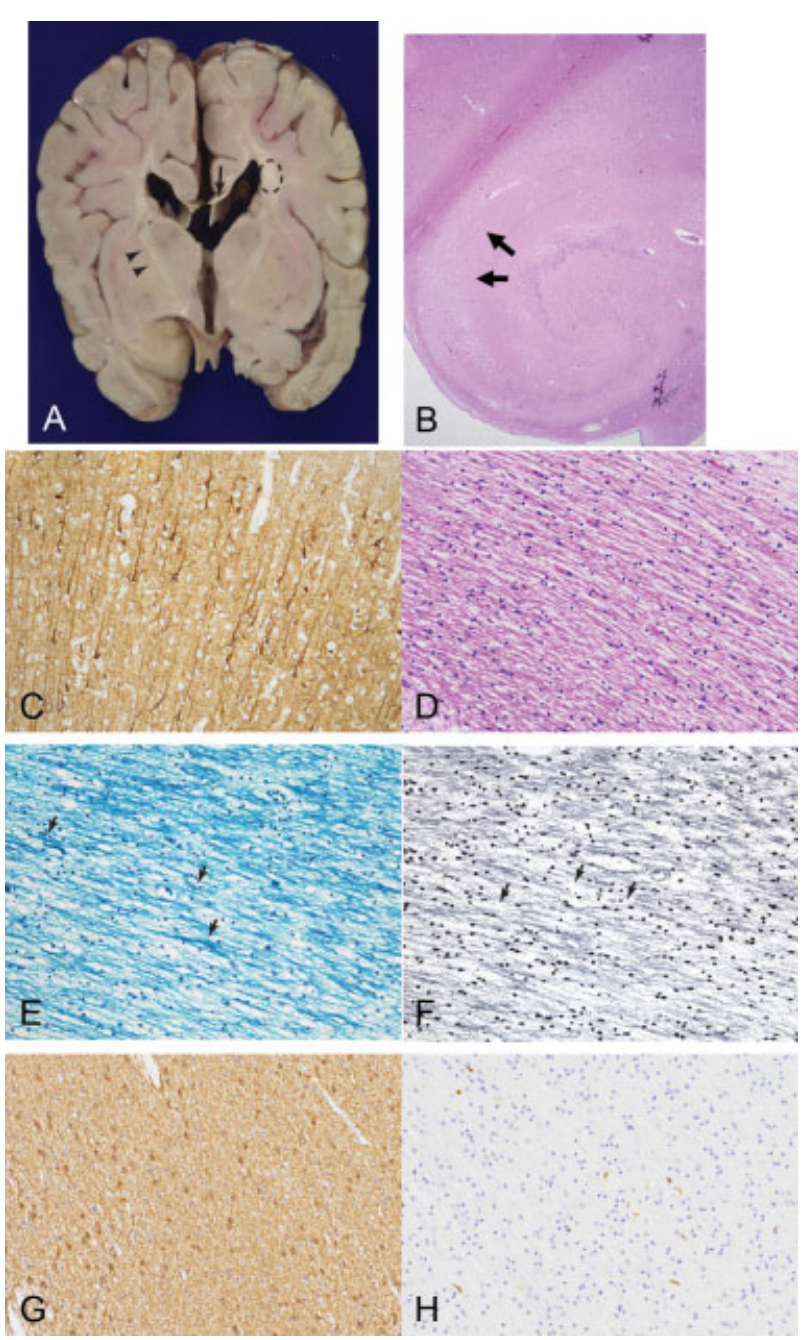

Fig. 2 Gross pathology and histopathology of the autopsy specimen. An extensive autopsy examination of the brain, eyes, and spinal cord was performed. Comprehensive immunohistochemical analysis of the different brain regions was examined and reported. A few representative images highlighting the unique findings of this case are presented. (A) Gross coronal section of the cerebral hemispheres, showing marked reduction in volume of centrum ovale (dotted circle) and width of the corpus callosum (arrow). The internal capsule is of relative normal size (arrow heads). White matter appears myelinated. (B) Hematoxylin and eosin stained section of the hippocampus demonstrating neuronal loss (arrows). (C) Neurofilament protein staining of frontal cortex highlighting pyramidal neurons. (D-F) Histological images from sections through the corpus callosum, hematoxylin and eosin (D). A paucity of myelinated fibers is present on Klüver stain (E, arrows). The same area of corpus callosum shows fragmented axons on Bodian stain ( $F$, arrows). Lack of astrogliosis and macrophage response was highlighted by glial fibrillary acidic protein $(G)$ and CD163 $(H)$ stains, respectively. Image $B$ at $25 \times$ magnification, image $C$ at $100 \times$, and images $D-H$ at $200 \times$ magnification.

field was replaced by either thinly myelinated axons or by circular myelin-like membranous profiles of diameters ranging from 3 to 15 microns that lacked axons (-Fig. 3A-C, arrows). Processes of glial cells containing intermediate filaments, as in reactive gliosis, were not seen. Similarly, macrophages containing myelin debris were not seen (see both - Fig. 3). The integrity of axons was difficult to evaluate, due to fixation-preservation issues. However, axons with
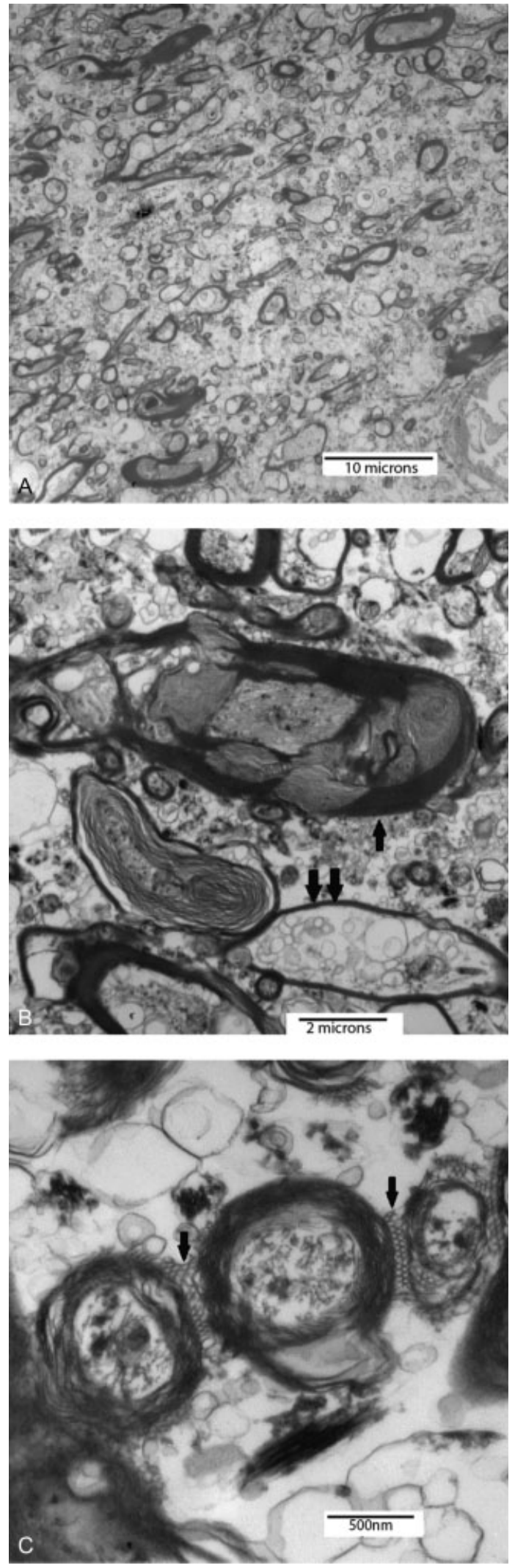

Fig. 3 Electron microscopic images of white matter at low power (1800x; A) and higher magnification $(7100 x$; $B)$. There is preservation of the neurofilaments in the myelinated axon. Single arrow (B) shows disruption of myelin structure and areas of "myelin figures" and amorphous material. The double arrow demonstrates myelinated processes in which the axon is replaced by myelin figures, likely due to fixation. Unusual "honey-comb" like profiles between myelinated axons are shown in (C) (28,000x magnification). 
intact neurofilaments were seen surrounded by disrupted myelin and amorphous deposits (-Fig. 3C).

Unusual "honey-comb" or "zipper-like" profiles were seen between adjacent myelinated axons ( - Fig. 3C). The length of these unusual profiles was $250 \mathrm{~nm}$ on average (-Fig. 3C), while each unit composing the "honey-comb" measured 25 to $50 \mathrm{~nm}$. These unusual profiles were restricted to areas of adjacent myelin sheaths and might represent artifact of fixation or an unusual "lesion" of myelin associated with polyol dysregulation.

Oligodendrocytes, identified by nuclear size and chromatin pattern, had cytoplasm which was virtually empty of the usual organelles, that is, mitochondria, Golgi apparatus, and microtubules. However, considering the known sensitivity of oligodendrocytes to fixation and processing artifact, it is difficult to interpret this observation.

\section{Discussion}

Primary disorders of polyol metabolism were first described in 1999 by van der Knapp et al in a boy who developed epilepsy at age 4 years in the setting of moderate developmental delays. The seizures became intractable and the patient followed a regressive course characterized by deterioration of speech, vision, and motor functions. The MRI showed diffuse T2 prolongation in white matter. ${ }^{17}$ Subsequent screens for polyols in urine, blood, and cerebrospinal fluid in over 100 patients led to discovery of four additional patients with abnormalities of polyols. ${ }^{8,16}$ Two of these four children had neonatal onset of epilepsy and profound developmental disabilities very similar to our patient. The urine polyol findings as well as their imaging characteristics were also similar to those observed in our reported child. The other two patients described by van der Knapp et al had completely different phenotypes: one had childhood onset epilepsy without developmental regression and the other hepatosplenomegaly without a neurological component. ${ }^{16}$ Subsequently, several disorders linked to polyol dysmetabolism have been described, with the genetics of a few described. The neuropathology of this condition has not been presented. Here, we describe novel neuropathology in a patient with a profound epileptic encephalopathy with abnormal polyol metabolites in two samples, 2 years apart.

Detailed postmortem findings of our 5-year-old child with early-onset intractable epilepsy, profound hypotonia, lack of meaningful development and MRI, and pathological documentation of paucity of white matter suggest that polyol dysmetabolism interferes with myelination due to abnormalities in oligodendrocytes. Polyol dysmetabolism was speculated, in a review of leukodystrophies by Schiffmann and van der Knaap, to cause a primary myelin disorder. ${ }^{18}$ The finding of hypomyelination is suggested by no evidence of myelin breakdown, specifically, the absence of macrophages on light microscopy, lack of expression of CD163 (a macrophage marker), and no gliosis. The metabolic consequences of polyol build up are not known, but the ultrastructural finding of abnormal morphology of oligodendroglia, the cells responsible for myelin formation, suggests that increasing levels of polyol metabolites are toxic for such cells. With diffuse neuronal loss also present on neuropathology, we cannot state if the effects of polyol dysmetabolism are somewhat specific for oligodendroglia, with secondary axonal and neuronal loss or if polyol accumulation is diffusely toxic to the brain and peripheral nervous system.

One unique pathological finding on the ultrastructural studies was the presence of "honey-comb" or "zipper-like profiles." The significance of these structures remains to be defined as such structures have not been observed in CNS of any other individuals with hypomyelinating disorders. Although hypomyelination in the nervous system of our patient is similar in some respects to cerebral hypomyelination syndrome (vanishing white matter disease) and Pelizaeus-Merzbacher disease (PMD), it differs in that axons are present in those two disorders but were difficult to find in our patient. ${ }^{19-21}$ Moreover, PMD and vanishing white matter disease are both associated with astrocytosis and presence of fat-filled macrophages, conspicuously absent in our patient. However, demonstration of neuronal loss in retinal epithelium, Purkinje and basket cells of the cerebellum along with devastating absence of axons force consideration that the underlying pathology is more complex than one caused only by malfunction of oligodendroglia.

The pathophysiological changes that occur with accumulation of polyols are not well-understood. Studies in hyperglycemia, diabetic polyneuropathy, and galactosemia suggest that accumulation of polyols within cells results in increased intracellular osmoles bringing excessive water into tissue resulting in tissue swelling and cell death., ${ }^{9,10,12}$ In diabetics and patients with galactosemia, this mechanism is thought to explain the peripheral neuropathy, cataracts, and retinopathy. ${ }^{9,10,12,22}$ on the basis of the mechanism proposed for diabetes and galactosemia, we hypothesize that greater accumulation of polyols occurs within premyelinating oligodendrocytes or Schwann cells resulting in the absence of myelin found on serial MRIs and confirmed postmortem in the CNS of our patient.

It is possible that the very low carbohydrate content of the ketogenic diet used to treat this child's epilepsy delayed the accumulation of the polyols in the urine. Therefore, the initial tests obtained at age 2 years were just mildly elevated, and it was not until they were repeated at age 4 years that a diagnosis was made. It is also possible that the elevated urine polyols are completely unrelated, but the clinical phenotype fits within what is currently known as the "polyol disorder umbrella." The pathology of such a case has not before been reported, and it is unique and interesting.

There are a few limitations of this case report that should be noted. First, whole exome sequencing was not performed, and only single gene testing for genes known to cause epilepsy at the time was sent. Unfortunately, this patient passed prior to the large-scale use of massively parallel sequencing as a major method for diagnosis in infants with unknown conditions, so we are unable to perform this analysis on this patient. A second limitation, while not typically performed clinically, is the lack of direct CNS proof of altered polyol metabolism, either from cerebrospinal fluid polyol levels or direct tissue measurements. 
A third limitation is the polyol elevations in our patient were not as high as some articles have reported. However, the levels in our patient were above the normal range and the pattern of elevation was consistent between two samples 2 years apart. Even with these limitations, as this is the first case of a profound epileptic encephalopathy with possible polyol disorder with a postmortem examination, it is clear that much remains to be learned regarding the pathophysiology, molecular pathways, and genetics in the pathogenesis of this disorder.

\section{Conflict of Interest}

None declared.

\section{Acknowledgments}

The authors would like to thank the family of the patient for agreeing to autopsy and contributing to medical knowledge. We would like to acknowledge Dr. Nick Gonatas, who performed the EM for this paper who unfortunately passed away before the manuscript could be submitted.

\section{References}

1 O'Brien MM, Schofield PJ, Edwards MR. Polyol-pathway enzymes of human brain. Partial purification and properties of sorbitol dehydrogenase. Biochem J 1983;211(01):81-90

2 Joost HG, Thorens B. The extended GLUT-family of sugar/polyol transport facilitators: nomenclature, sequence characteristics, and potential function of its novel members (review). Mol Membr Biol 2001;18(04):247-256

3 Demirbas D, Coelho AI, Rubio-Gozalbo ME, Berry GT. Hereditary galactosemia. Metabolism 2018;83:188-196

4 Vaca G, Ibarra B, Bracamontes M, et al. Red blood cell sorbitol dehydrogenase deficiency in a family with cataracts. Hum Genet 1982;61(04):338-341

5 Wamelink MM, Ramos RJ, van den Elzen AP, et al. First two unrelated cases of isolated sedoheptulokinase deficiency: a benign disorder? J Inherit Metab Dis 2015;38(05):889-894

6 Onkenhout W, Groener JE, Verhoeven NM, Yin C, Laan LA. L-Arabinosuria: a new defect in human pentose metabolism. Mol Genet Metab 2002;77(1-2):80-85

7 Verhoeven NM, Wallot M, Huck JH, et al. A newborn with severe liver failure, cardiomyopathy and transaldolase deficiency. J Inherit Metab Dis 2005;28(02):169-179
8 Huck JH, Verhoeven NM, Struys EA, Salomons GS, Jakobs C, van der Knaap MS. Ribose-5-phosphate isomerase deficiency: new inborn error in the pentose phosphate pathway associated with a slowly progressive leukoencephalopathy. Am J Hum Genet 2004;74(04): 745-751

9 Cooper ME. Pathogenesis, prevention, and treatment of diabetic nephropathy. Lancet 1998;352(9123):213-219

10 Lee AY, Chung SK, Chung SS. Demonstration that polyol accumulation is responsible for diabetic cataract by the use of transgenic mice expressing the aldose reductase gene in the lens. Proc Natl Acad Sci U S A 1995;92(07):2780-2784

11 Regenold WT, Phatak P, Makley MJ, Stone RD, Kling MA. Cerebrospinal fluid evidence of increased extra-mitochondrial glucose metabolism implicates mitochondrial dysfunction in multiple sclerosis disease progression. J Neurol Sci 2008;275(1-2):106-112

12 Berry GT. The role of polyols in the pathophysiology of hypergalactosemia. Eur J Pediatr 1995;154(07, Suppl 2):S53-S64

13 Shetty HU, Schapiro MB, Holloway HW, Rapoport SI. Polyol profiles in Down syndrome. myo-inositol, specifically, is elevated in the cerebrospinal fluid. J Clin Invest 1995;95(02):542-546

14 Regenold WT, Hisley KC, Obuchowski A, Lefkowitz DM, Marano C, Hauser P. Relationship of white matter hyperintensities to cerebrospinal fluid glucose polyol pathway metabolites-a pilot study in treatment-resistant affective disorder patients. J Affect Disord 2005;85(03):341-350

15 Moolenaar SH, van der Knaap MS, Engelke UF, et al. In vivo and in vitro NMR spectroscopy reveal a putative novel inborn error involving polyol metabolism. NMR Biomed 2001;14(03):167-176

16 Huck JH, Verhoeven NM, van Hagen JM, Jakobs C, van der Knaap MS. Clinical presentations of patients with polyol abnormalities. Neuropediatrics 2004;35(03):167-173

17 van der Knaap MS, Wevers RA, Struys EA, et al. Leukoencephalopathy associated with a disturbance in the metabolism of polyols. Ann Neurol 1999;46(06):925-928

18 Schiffmann R, van der Knaap MS. Invited article: an MRI-based approach to the diagnosis of white matter disorders. Neurology 2009;72(08):750-759

19 Costello DJ, Eichler AF, Eichler FS. Leukodystrophies: classification, diagnosis, and treatment. Neurologist 2009;15(06):319-328

20 Mar S, Noetzel M. Axonal damage in leukodystrophies. Pediatr Neurol 2010;42(04):239-242

21 Bugiani M, Boor I, van Kollenburg B, et al. Defective glial maturation in vanishing white matter disease. J Neuropathol Exp Neurol 2011;70(01):69-82

22 Forcier NJ, Mizisin AP, Rimmer MA, Powell HC. Cellular pathology of the nerve microenvironment in galactose intoxication. J Neuropathol Exp Neurol 1991;50(03):235-255 